\title{
Cuarenta ańos de activismo empresarial antioqueño en los procesos de paz en Colombia (1980-2020)
}

\author{
Germán Darío Valencia-Agudelo (10 ${ }^{a}$
}

-Exordio. -I. De las primeras iniciativas de paz hasta los acercamientos con las FARC-EP (1980-2010). -II. El rol activo del sector empresarial en el proceso de paz con las FARC-EP (2010-2020). -Colofón. -Referencias.

Primera versión recibida el 30 de mayo de 2020; versión final aceptada el 1 de junio de 2020

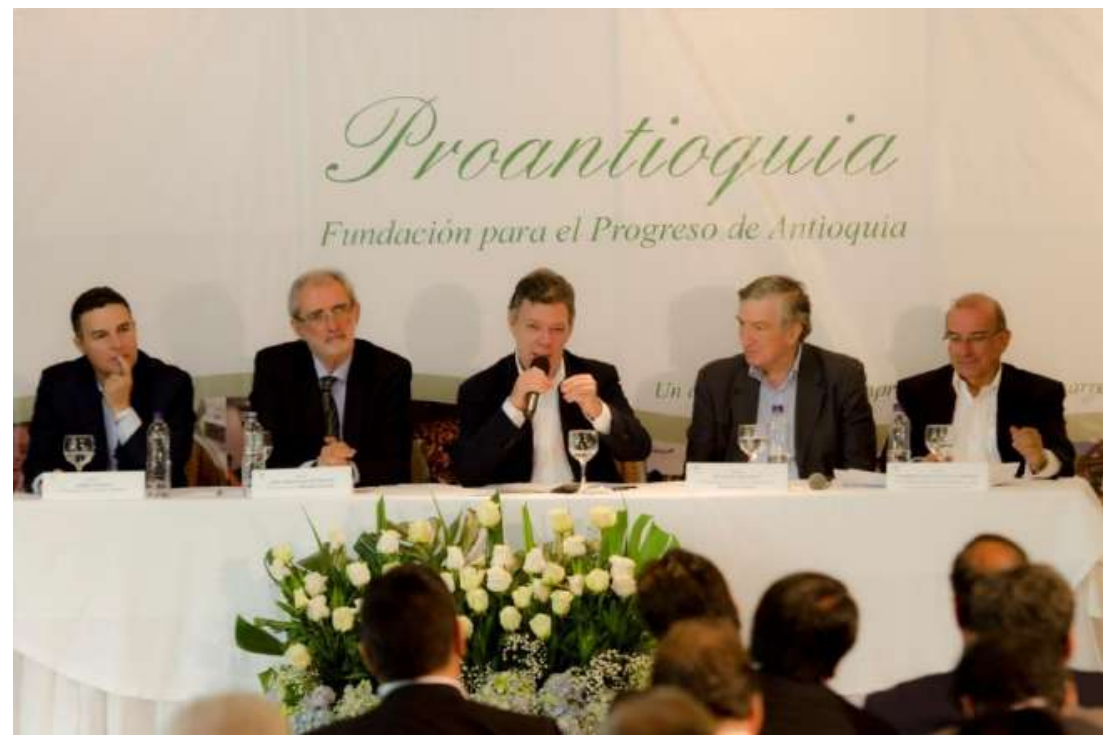

Fuente: Proantioquia (2013) ${ }^{1}$.

a Germán Darío Valencia Agudelo: investigador y miembro del Grupo Hegemonía, guerras y conflictos, del Instituto de Estudios Políticos de la Universidad de Antioquia, Colombia. Dirección postal: Instituto de Estudios Políticos, oficina 14-209, Calle 70 No. 52-21, Medellín, Colombia. Dirección electrónica: german.valencia@udea.edu.co https://orcid.org/0000-0002-6412-6986

https://doi.org/10.17533/udea.le.n93a342251

\section{(cc) BY-NC-SA}

Este artículo y sus anexos se distribuyen por la revista Lecturas de Economía bajo los términos de la Licencia Creative Commons Atribución-NoComercial-CompartirIgual 4.0. https://creativecommons.org/licenses/by-nc-sa/4.0/ 
Valencia-Agudelo: Cuarenta años de activismo empresarial antioqueño en los procesos de paz...

\section{Exordio}

Es curioso, el momento del nacimiento de la revista Lecturas de Economía coincide con el surgimiento del más reciente período de los procesos de paz en Colombia (1980-2020). Justo en enero de 1980, cuando los profesores del Departamento de Economía de la Universidad de Antioquia estaban discutiendo el nombre que llevaría nueva revista y el contenido de sus primeros números (Velázquez, 2015, p. 257), el país se encontraba atento a la respuesta que el presidente de la República, Julio César Turbay Ayala (1978-1982), le daría a la propuesta de Carlos Lleras Restrepo de crear una organización para que explorara una salida negociada a la guerra. La propuesta, que fue aceptada y dio origen a la primera Comisión de Paz —el 8 de octubre de 1981, mediante el Decreto 2761—, sería el punto de inicio de una larga y fecunda experiencia de procesos de paz en Colombia, en la que se cuentan hasta la fecha por lo menos 58 transacciones entorno a la paz (Valencia, 2019, p. xi).

Este discurrir histórico de búsqueda de la paz en Colombia cumple en 2020 cuarenta años. En él han participado muchos actores nacionales e internacionales, pero en esta nota se quiere resaltar el papel activo del sector empresarial y, de manera puntual, el que ha tenido el sector empresarial antioqueño en esta larga historia. Historia que coincide con la de la revista Lecturas de Economía, que en este número 93 celebra sus cuarenta años. En estas décadas, la revista ha mostrado, en repetidas ocasiones, un interés por la cara opuesta a la paz: me refiero a la guerra o, como los economistas políticos lo han llamado, el conflicto armado interno (Valencia, 2006). En varios números, Lecturas de Economía ha publicado fecundos textos sobre el

1 Imagen tomada de la XLII Asamblea de Proantioquia, realizada el martes 9 de abril de 2013, donde se aprobó una proposición en la que el empresariado antioqueño expresó su apoyo a los esfuerzos que el Gobierno de Juan Manuel Santos realizaba en las conversaciones con delegados de las FARC en La Habana, Cuba (Proantioquia, 2013). En la imagen, de izquierda a derecha, están: Aníbal Gaviria Correa (quien fuera alcalde de Medellín, 20122015), Juan Sebastián Betancur (presidente de Proantioquia), Juan Manuel Santos (presidente de la República de Colombia, 2010-2018), Nicanor Restrepo Santamaría (presidente de la junta directiva de Proantioquia) y Humberto de la Calle (Jefe Negociador de la delegación del Gobierno de Colombia en la Mesa de Conversaciones de La Habana). 
conflicto y su relación con la economía: en unos, analizando a los actores armados (por ejemplo, en Castillo \& Salazar, 2007) y, en otros, el mercado ilegal de las drogas (Ortiz, 2003, 2009; Gutiérrez \& Tobón, 2019).

Quiero destacar en la historia de la revista un trabajo publicado en 1997 por Jesús Antonio Bejarano (justo dos años antes de su trágica muerte, el 15 de septiembre de 1999), titulado "Inseguridad, violencia y actividad económica", donde realiza una profunda reflexión sobre la relación entre el conflicto armado y la economía colombiana. "Chucho" Bejarano, como lo llamaban sus amigos, fue un brillante economista y fecundo pensador, además uno de los seres más comprometidos con la paz en el país tanto como ciudadano como académico, a tal punto que se le considera el fundador en Colombia de los estudios científicos de la paz (Bejarano, 2011). Fungió desde el Gobierno de Virgilio Barco (1986-1990) como experto en la resolución de conflictos, y se desempeñó como consejero de paz y negociador con las guerrillas durante el Gobierno de César Gaviria (1990-1994). En síntesis, Bejarano tuvo una hermosa combinación: fue un profundo académico y economista ejemplar y, a la vez, un abanderado y gestor de la paz y del diálogo en el país.

En esta nota se quiere destacar el papel crucial que han cumplido y vienen cumpliendo los empresarios y los gremios del departamento de Antioquia, Colombia, en la construcción de la paz del país. Para mostrarlo, en el texto se hace uso de la cronología histórica, por lo que se parte de las primeras iniciativas de paz al inicio de la década de 1980 y se avanza en la narración hasta los acercamientos que se dieron con las Fuerzas Armadas Revolucionarias de Colombia-Ejército del Pueblo (FARC-EP) en 2010. Luego, en la segunda parte, se analiza el rol que tuvo el sector empresarial antioqueño en el proceso de paz con esta guerrilla y que ha tenido en su actual implementación. Para finalmente, a modo de colofón, realizar dos propuestas que, de ser acogidas, lograrían impulsar mucho más el activismo empresarial entorno a la paz.

\section{De las primeras iniciativas de paz hasta los acercamientos con las FARC-EP (1980-2010)}

El Gobierno Turbay aceptó en 1981 la invitación para buscar una salida negociada al conflicto armado, pero fue su sucesor, el presidente Belisario 
Valencia-Agudelo: Cuarenta años de activismo empresarial antioqueño en los procesos de paz...

Betancur (1982-1986), quien realmente ejecutó la decisión gubernamental. Durante su mandato, Betancur reformó la primera Comisión de Paz y creó otras organizaciones encargadas de impulsar el acercamiento, el diálogo y la implementación. Entre las primeras organizaciones creadas tenemos los Altos Comisionados para la Paz (Decreto 240 del 4 de febrero de 1983) en las que el presidente quiso involucrar directamente al sector empresarial en el logro de la paz.

Betancur nombró al líder empresarial antioqueño Nicanor Restrepo Santamaría entre sus representantes (Rettberg \& Aceros, 2013, p. 20), pues quería rodearse de personas expertas en administrar dineros y convertirlos en los "Gerentes de la Paz" (Morales, 1983, citado en Arias, 2008, p. 14). De allí que a los Altos Comisionados para la Paz se les haya asignado labores más ejecutivas, como las de "coordinar, ejecutar y evaluar el avance de los programas de microempresas de rehabilitación, dotación de tierras y créditos, educación y salud" (Valencia, 2019, p. 167). Luego, cuando el presidente logró firmar el "Acuerdo de La Uribe" con las FARC-EP, el 28 de marzo de 1984, creó la Comisión de Verificación, para la cual llamó de nuevo a los empresarios antioqueños Nicanor Restrepo y Juan Sebastián Betancur Escobar a integrar esta organización especializada (Rettberg \& Aceros, 2013, p. 20).

Esta apuesta por la paz durante el decenio de 1980 no se dio solo a través de empresarios, sino también de gremios (Kalmanovitz, 1991, p. 202). La Asociación Nacional de Industriales (ANDI), que es uno de los gremios más importantes del sector privado, en sus diversas asambleas anuales sostuvo que la paz debería ser un propósito nacional. Por eso, "fue también la ANDI quien participó más activamente en los diálogos —a la postre fallidos — de La Uribe (Meta)" (Rettberg \& Rivas, 2012, p. 318). Esta apuesta por la paz se mantuvo durante el Gobierno de Virgilio Barco, cuando el presidente se convirtió en uno de los miembros de las Mesas de Trabajo por la Paz y la Reconciliación Nacional.

Más tarde, en la década siguiente, en medio de la fiesta que produjo la desmovilización de ocho agrupaciones armadas, entre 1990 y 1994, de nuevo los empresarios antioqueños ratificaron su apoyo a la realización de 
la paz en Colombia. Esta vez, en la Comisión Asesora de Reinserción ${ }^{2}$, cuyo objetivo fue asesorar al Gobierno en temas de empleo y búsqueda de fórmulas para la reintegración a la vida civil de los guerrilleros desmovilizados (García, 1992). De esta Comisión, integrada en su totalidad por empresarios y líderes gremiales colombianos, hicieron parte Andrés Echavarría (miembro de la junta directiva de la Fundación Corona), Isaac Jimmy Mayer (industrial del sector textil), Nicanor Restrepo (quien actuaba en nombre del Grupo Empresarial Antioqueño - GEA-) y Carlos Upegui (gerente de Empresas Públicas de Medellín y presidente de Coltejer) (Rettberg \& Aceros, 2013, p. 39).

Durante el gobierno de Ernesto Samper (1994-1998), los empresarios apoyaron la propuesta presidencial de Diálogo Útil (Villarraga, 2009). Los grandes empresarios antioqueños, en especial los vinculados con el GEA, elaboraron una carta en junio de 1997, donde manifestaron al presidente Samper su respaldo a la búsqueda de una salida negociada y le solicitaron que "como jefe de Estado, proceda a dar los pasos necesarios para iniciar y llevar adelante el proceso de paz" (Forero, 1997), para el cual ofrecieron su apoyo y colaboración.

Finalmente, la mayor presencia e incidencia del sector empresarial antioqueño en la paz durante el período analizado se dio en las negociaciones realizadas entre las FARC-EP y Andrés Pastrana (1998-2002). Durante este Gobierno los empresarios se convirtieron en actores estratégicos en la política de paz nacional, por eso "siempre hicieron parte del equipo negociador" (Rettberg \& Aceros, 2013, p. 59). Y quién más participó en estas negociaciones fue Nicanor Restrepo Santamaría, el cual, debido a su amplia experiencia, integró la Mesa Nacional de Diálogo y Negociación, una instancia decisoria del proceso de negociación y la encargada de elaborar el Acuerdo de La Machaca, Caquetá, con la Agenda común para el cambio hacia una nueva Colombia.

2 Decretos 2198 y 2199 del 23 septiembre de 1991 y el Decreto 2207 del 25 de septiembre del mismo año. 
Valencia-Agudelo: Cuarenta años de activismo empresarial antioqueño en los procesos de paz...

\section{El rol activo del sector empresarial en el proceso de paz con las FARC-EP (2010-2020)}

En agosto de 2010 inicia el otro momento importante de procesos de paz en el país. Luego de ocho años de intenso conflicto armado durante el mandato de Álvaro Uribe, el Gobierno de Juan Manuel Santos (20102018) abre nuevamente la puerta a una salida negociada a la guerra. Durante esta última década los empresarios antioqueños de nuevo han mantenido un activismo en los procesos de paz. Ha sido una década donde se ha observado una participación e incidencia de los empresarios en las fases de acercamiento, negociación e implementación. El sector empresarial ha participado de manera decidida en los acercamientos, como mediador con Henry Acosta Patiño; en las negociaciones, apoyando el proceso y brindando insumos para ellas; y en el posacuerdo, participando en la reincorporación económica y social de excombatientes.

En la fase pública de negociaciones, que duró cuatro años (de noviembre de 2012 a noviembre de 2016), los empresarios antioqueños tuvieron contacto directo con la Mesa de Conversaciones de La Habana. Por un lado, mediante la presencia de algunos empresarios de Antioquia que visitaron en repetidas ocasiones las comisiones delegadas; así por ejemplo, en 2015, los representantes David Bojanini del Grupo Sura, Carlos Raúl Yepes del Grupo Bancolombia, Carlos Gallego de Nutresa y el rector de la Universidad Eafit, Juan Luis Mejía, se reunieron con los negociadores del Gobierno y la guerrilla para hablar sobre la construcción de la paz, lo que sirvió para ratificar el respaldo empresarial antioqueño a las negociaciones (Portafolio, 2015). Por otro lado, los empresarios paisas tuvieron representantes directos y permanentes en la Mesa de Conversaciones, como Luis Carlos Villegas, que, aunque representaba al Gobierno, se tomó como la ficha de los empresarios en la Mesa, pues tuvo voz y voto - poder plenipotenciario-. Villegas, que se desempeñó por dieciocho años como presidente de la ANDI y presidente del Consejo Gremial Nacional y Consejo Industrial Andino, sirvió de negociador durante un año en la Mesa (noviembre 2012 a noviembre 2013); además también se tuvo en el equipo negociador a Gonzalo Restrepo, expresidente del Grupo Éxito. 
Igualmente, el sector empresarial antioqueño, en variadas ocasiones, incidió externamente en las negociaciones, manifestando públicamente su apoyo al Gobierno nacional durante el tiempo que duraron los diálogos. Estas expresiones de apoyo comenzaron en 2013, cuando los empresarios antioqueños dieron su apoyo al proceso de paz: "El organismo empresarial más importante de Antioquia (Proantioquia), anunció públicamente su respaldo a los diálogos que se adelantan en La Habana, Cuba" (Semana, 2013). Un año después, en junio de 2014, el apoyo fue ratificado con un grupo aún mayor de empresarios (80), quienes hablaron de la confianza en el proceso de paz y consideraron que era fundamental que, en un nuevo periodo presidencial, se continuara con los programas de construcción de paz (El Tiempo, 2014). Y de nuevo en 2015 se escucharon voces de apoyo empresarial en distintos eventos y foros donde se presentaban los avances en las negociaciones de La Habana.

Finalmente, a partir del 25 noviembre de 2016, con la firma del Acuerdo final para la terminación del conflicto y la construcción de una paz estable y duradera, el sector empresarial antioqueño ha logrado consolidar su idea de activismo en torno a la paz. Lo está haciendo de varias formas: primero, vinculándose y apoyando los procesos de reintegración social y económica. Por ejemplo, el Grupo Éxito ha diseñado un programa de "Inclusión Laboral" con el que ha incorporado en su planta de personal a desmovilizados y a sus familiares (ANDI, 2019); por su lado, ISA, con su programa Prodepaz en el Oriente Antioqueño, ha financiado y asistido proyectos y emprendimientos de desarrollo en zonas afectadas por el conflicto (Mustafá, 2017, p. 31). Segundo, las empresas paisas han apoyado la reincorporación colectiva de la exguerrilla con la donación de tierras a los reincorporados, caso el lote de 270 hectáreas en Llano Grande, municipio de Dabeiba, entregado por las empresas antioqueñas Postobón, Grupo Sura, Grupo Nutresa, Bancolombia, Grupo Argos, Corbeta y la Fundación Fraternidad Medellín (Forero, 2019). Finalmente, han participado en la construcción de paz invirtiendo en las zonas más afectadas por el conflicto —ZOMAC—. En estos territorios algunas empresas paisas vienen realizando inversiones y, a cambio, se les 
Valencia-Agudelo: Cuarenta años de activismo empresarial antioqueño en los procesos de paz...

reduce impuestos ${ }^{3}$ (Fundación Ideas para la Paz —FIP_, 2017, p. 41); entre dichas empresas están Corona, Arturo Calle, Crepes \& Waffles, la Compañía Nacional de Chocolates, EPM e ISA.

En síntesis, la revisión de la historia reciente de Colombia permite constatar que, desde 1980 hasta 2010, el sector empresarial antioqueño ha buscado asumir una presencia cada vez mayor en la consecución de la paz. Desde comienzos de este período se observa un importante número de experiencias de participación del sector empresarial en las dinámicas de los procesos de paz y se ha visto cómo, a través de algunos representantes, han logrado apoyar al Estado en los acercamientos y el inicio de los diálogos de paz, ofreciéndose de enlace de comunicación y creando confianza entre las partes para facilitar el inicio de los diálogos, sirviendo de mediador silencioso pero eficiente y proponiendo en las hojas de ruta temas claves para el país y la ciudadanía.

En la fase de negociación se vio a los empresarios realizando foros, seminarios y encuentros donde manifestaron sus intereses de incidir en ciertos temas en la negociación; integrando la comisión de paz con la que se han explorado las salidas negociadas al conflicto armado interno; participando de manera directa, mediante delegados plenipotenciarios, en las comisiones de negociación; y promoviendo por fuera de la mesa de negociación una opinión pública favorable, por lo que han cumplido un papel de legitimador del proceso de paz. De igual forma, en los posconflictos han apoyado los programas de reintegración económica y social de los excombatientes ofertándoles empleos a ellos y a las víctimas del conflicto; también han apadrinado las pequeñas empresas de proyectos productivos tanto para exguerrilleros como para comunidades que habitan en territorios afectados por la violencia; han apoyado al Gobierno en la implementación de programas de desarrollo; han aprovechado responsablemente los incentivos tributarios

3 El programa Obras por Impuestos es una iniciativa gubernamental que busca el apoyo del sector privado en la implementación del Acuerdo Final. Fue creado en 2016 mediante la Ley 1819 y reglamentado por el Decreto 1915 de 2017. En el cuarto informe del Instituto Kroc de Estudios Internacionales de Paz, (2020, p. 81), informa que "el programa aprobó 65 proyectos durante el 2019 por un valor de 290 mil millones de pesos, los cuales beneficiaron a la mayoría de los municipios PDET'. 
para la paz, recibiendo la reducción de impuestos a cambio de las inversiones que hacen en la construcción de paz; y han comprado los servicios, productos y cultivos de las cooperativas de exguerrilleros.

\section{Colofón}

Por último, se ha querido dejar para el cierre de esta nota un par de propuestas, a modo de colofón, para que se continúe trabajando en la construcción de la paz desde el activismo empresarial, considerando que esta labor debe realizarse en un entorno político de posacuerdo y con la presencia de Grupos Armados Organizados — GAO_ (por ejemplo, el Ejército de Liberación Nacional _ELN_), por lo que se hace más urgente continuar avanzando en acciones y prácticas que señalan las maneras como las empresas y los gremios deben participar en la construcción de paz. En este sentido, se reconoce el papel clave que juegan los empresarios en la construcción de la paz.

La primera propuesta es continuar trabajando en las zonas mas afectadas por el conflicto, en aquellos territorios excluidos y marginados que han estado alejados de las dinámicas del desarrollo social y económico del centro del país. En estos territorios está la población que más se ha visto afectada por el accionar de los fusiles durante muchas décadas. Es conveniente para el país insistir en la idea de trabajar en los lugares devastados por la guerra y así romper el círculo vicioso que existe entre violencia y pobreza. Ahora que la paz con las FARC-EP es una realidad se requiere trabajar en proyectos que beneficien a toda la sociedad. La propuesta que se le hace al sector empresarial antioqueño, extensivo al empresariado nacional y a las empresas multinacionales, es trabajar para la implementación de programas de reconstrucción socioeconómica. Se debe insistir, por un lado, en un aumento de inversiones en las regiones más afectadas por el conflicto, tanto las ZOMAC como los territorios donde se diseñaron los Programas de Desarrollo con Enfoque Territorial _-PDET_- Valencia \& Restrepo, 2020). Es urgente que el sector empresarial se haga sentir en todas las regiones, apoyando el desarrollo económico y social, buscando "atraer y facilitar el acceso al capital, así como proveer asistencia técnica y financiera” (Mustafá, 
Valencia-Agudelo: Cuarenta años de activismo empresarial antioqueño en los procesos de paz...

2017, p. 26). Es necesario trabajar en estos territorios con el objetivo de sentar bases sólidas para construir territorios de paz.

La segunda propuesta, que complementa la anterior, es que se busque impulsar la implementación del Acuerdo Final y el seguimiento al cumplimiento de los compromisos que se han pactado. El Acuerdo Final debe verse como un programa de desarrollo para el país. De allí que cada uno de los puntos que contiene debe impulsarse para su ejecución. De forma concreta, "[...] es necesario que los empresarios asuman el liderazgo sobre las reformas que se vienen, de manera tal que estas se hagan teniendo en cuenta su punto de vista, y no a sus espaldas" (Gallego et al., 2016, p. 36). El sector empresarial debe establecer un plan de acción frente a cada uno de los puntos del Acuerdo Final: en la defensa de programas que incluyan políticas sociales — como en educación, salud y servicios públicos- para el sector rural en el punto uno relativo al Desarrollo Rural Integral. Además, es necesario que los empresarios asuman la tarea de acompañar a diversas organizaciones en hacer seguimiento al cumplimiento de la acordado en La Habana. Su experiencia ayudaría a que fluya información clara y precisa sobre la manera como se viene implementando el Acuerdo. Se debe en este aspecto tener un sector empresarial activo, crítico y vigilante de la implementación. No se puede dejar el papel de seguimiento solo a la Comisión de Seguimiento, Impulso y Verificación a la Implementación del Acuerdo Final, _CSIVI-, la empresa debe aportar con sus equipos de investigación al seguimiento a la implementación, para que vigilen y hagan cumplir los acuerdos pactados.

Es conveniente que la empresa se pregunte por su rol en el posacuerdo, que piense en "las agendas de las empresas desde sus áreas de responsabilidad social y sostenibilidad" (Gallego et al., 2016, p. 33). En definitiva, se requiere que las empresas y gremios antioqueños ayuden en este momento decisivo para el país en la recuperación de la economía y en la estabilización social y política de esta sociedad en un entorno de posacuerdo y con la presencia de GAOs que exigen un compromiso de este sector en la construcción de paz. 


\section{Referencias}

Asociación Nacional de Industriales (ANDI). (2019). La inclusión de víctimas y desmovilizados: una ventaja competitiva para las empresas en Colombia. Bogotá: Andi. http://www.andi.com.co/Uploads/Paper\%20Victimas \%20y\%20Desmovilizados\%20Lectura.pdf

Arias, G. (2008). Una mirada atrás: procesos de paz y dispositivos de negociación del gobierno colombiano. (Working Papers, No. 4). Fundación Ideas para la Paz. https://www.files.ethz.ch/isn/152333/mirada_atras_web.pdf

Bejarano, J. (1997). Inseguridad, violencia y actividad económica. Lecturas de Economía, 47, 7-24. https://doi.org/10.17533/udea.le.n47a4934

Bejarano, J. (2011). Antología Jesús Antonio Bejarano. Volumen 2. Estudios de Paz. Colección Obra Selecta. Bogotá: Universidad Nacional.

Castillo, M. \& Salazar, B. (2007). Alianzas y política: un juego entre agentes civiles y armados. Lectura de Economía, 67, 71-98. https://doi.org/10.1 7533/udea.le.n67a2021

Forero, S. (2019, noviembre 17). La tierra que empresarios antioqueños entregarán a excombatientes de FARC. El Espectador. https://www.el espectador.com/colombia2020/territorio/la-tierra-que-empresarios-ant ioquenos-entregaran-excombatientes-de-FARC-articulo-891392

El Tiempo (2014, junio 11). 80 empresarios dan su respaldo a los procesos de paz. https://www.eltiempo.com/archivo/documento/CMS-14107875

Fundación Ideas para la Paz (FIP) (2017). El aporte empresarial a la paz y al desarrollo sostenible. Desafios y oportunidades. Bogotá: Fundación Ideas para la Paz.

Gallego, L., Gutiérrez, I., Osorio, D. \& Cortés, A. (2016). Los retos de los empresarios en la construcción de paz (Cuadernos de trabajo en Gobierno y Ciencias Políticas, No. 6). Universidad Eafit. https://repository.eafit.e du.co/bitstream/handle/10784/9658/cuadernos_trabajo_eafit_6.pdf?s equence $=1$ \&is Allowed $=\mathrm{y}$ 
Valencia-Agudelo: Cuarenta años de activismo empresarial antioqueño en los procesos de paz...

García, M. (1992). De La Uribe a Tlaxcala: Procesos de paz. Bogotá: Cinep.

Gutiérrez, I. \& Tobón, S. (2019). El gasto fiscal de la guerra contra los portadores de drogas ilícitas: una aproximación para Colombia. Lecturas de Economía, 91, 79-116. https://doi.org/10.17533/udea.le.n91a03

Instituto Kroc de Estudios Internacionales de Paz, Iniciativa Barómetro, Matriz de Acuerdos de Paz. (2020). "Tres años después de la firma del Acuerdo Final de Colombia: hacia la transformación territorial. Diciembre 2018 a noviembre 2019”. Informe 4. Bogotá, Colombia: Universidad de Notre Dame.

Kalmanovitz, S. (1991). Los gremios industriales ante la crisis. En Francisco Buitrago y Leon Zamosc (eds.). Al filo del caos: crisis politica en la Colombia de los años 80. Bogotá: Tercer Mundo Editores e IEPRI (Universidad Nacional).

Mustafá, H. (2017). El sector privado y la construcción de paz en Colombia: la mujer, un actor clave en la sostenibilidad de la paz. Bogotá: Pontificia Universidad Javeriana.

Ortíz, C. (2003). La guerra contra las drogas es contraproducente: un análisis económico de equilibrio general. Lecturas de Economía, 58, 47-68. https: //doi.org/10.17533/udea.le.n58a2579

Ortíz, C. (2009). War on Drugs is Counterproductive, Once Again. Lecturas de Economía, 71, 19-42. https://doi.org/10.17533/udea.le.n71a4813

Portafolio (2015, noviembre 12). Ocho grandes empresarios se reunieron en Cuba con las FARC. https://www.portafolio.co/negocios/empresas/ ocho-grandes-empresarios-reunieron-cuba-FARC-29178

Proantioquia (2013). Proposición en Asamblea de Afiliados. https://proantioqu ia.org.co/asamblea-de-afiliados-de-proantioquia-y-encuentro-con-el-p residente-juan-manuel-santos/

Rettberg, A. \& Aceros, J. (2013). La empresa de la paz. Negociaciones de paz y empresarios en Colombia (1982-2006) (Documentos del Departamento de Ciencia Política, No. 20). Universidad de los Andes. https://reposito 
rio.uniandes.edu.co/bitstream/handle/1992/24893/u619122.pdf?seque nce $=1$ \&isAllowed $=y$

Rettberg, A. \& Rivas, A. (2012). El sector empresarial y la construcción de paz en Colombia: entre el optimismo y el desencanto. En A. Rettberg (comp.). Construcción de paz en Colombia (pp. 305-348). Bogotá: Universidad de los Andes.

Semana (2013, junio 13). El apoyo a la paz de los empresarios antioqueños. Semana, 13 de abril de 2013. https://www.semana.com/economia/ar ticulo/el-apoyo-paz-empresarios-antioquenos/339826-3

Valencia, G. (2006). La economía frente al conflicto armado interno colombiano, 1990-2006. Perfil de Coyuntura Económica, 8, 140-174.

Valencia, G. (2019). Organizarse para negociar la paz. Medellín: Editorial Universidad de Antioquia.

Valencia, G. \& Restrepo, D. (2020). Democracia participativa y planeación del progreso en los Programas de Desarrollo con Enfoque Territorial. Campos en Ciencias Sociales, 8(1), 115-142.

Velásquez, J. (2015). Revista Lecturas de Economía (1980-2015): un devenir histórico. Lecturas de Economia, 83, 257-264. http://dx.doi.org/10.1753 3/udea.le.n83a09

Villarraga, Á. (Ed.). (2009). Insurgencia y cambio democrático, acuerdos de paz con el EPL y con otras agrupaciones armadas. En Biblioteca de la paz Vol. III. Acuerdos con el EPL, PRT, MAQL Y CRS, Diálogos con la CGSB. Bogotá: Fundación Cultura Democrática.

Cómo citar / How to cite this item:

Valencia-Agudelo, G. D. (2020). Cuarenta años de activismo empresarial antioqueño en los procesos de paz en Colombia (1980-2020). Lecturas de Economía, 93, 331-343.

https://doi.org/10.17533/udea.le.n93a342251 